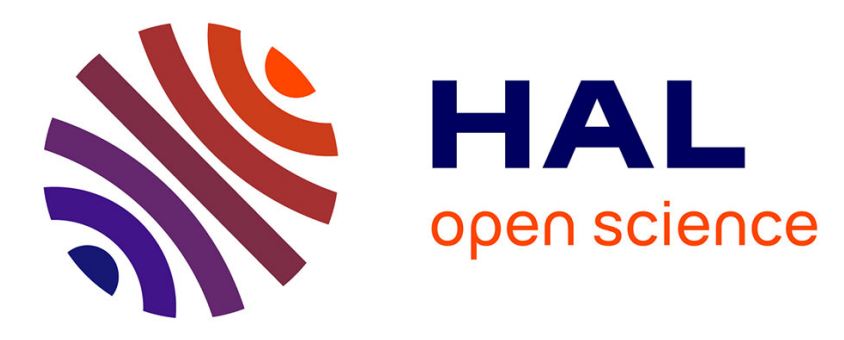

\title{
Risque lombaire pour les travailleurs exposés à des manipulations de charges: nécessité d'une prévention intégrée
}

Audrey Petit, Yves Roquelaure, Philippe Mairiaux, Arnaud Désarménien, Jean-Pierre Meyer, Sandrine Rousseau

\section{To cite this version:}

Audrey Petit, Yves Roquelaure, Philippe Mairiaux, Arnaud Désarménien, Jean-Pierre Meyer, et al.. Risque lombaire pour les travailleurs exposés à des manipulations de charges: nécessité d'une prévention intégrée. 33ème congrès national de médecine et santé au travail, Jun 2014, Lille, France. 75 (3), pp.S48, 2014, Archives des maladies professionnelles et de l'environnement. 10.1016/j.admp.2014.03.153 . hal-02928955

\section{HAL Id: hal-02928955 https://hal.science/hal-02928955}

Submitted on 14 Sep 2020

HAL is a multi-disciplinary open access archive for the deposit and dissemination of scientific research documents, whether they are published or not. The documents may come from teaching and research institutions in France or abroad, or from public or private research centers.
L'archive ouverte pluridisciplinaire HAL, est destinée au dépôt et à la diffusion de documents scientifiques de niveau recherche, publiés ou non, émanant des établissements d'enseignement et de recherche français ou étrangers, des laboratoires publics ou privés. 


\section{Risque lombaire pour les travailleurs exposés à des manipulations de charges : nécessité d'une prévention intégrée.}

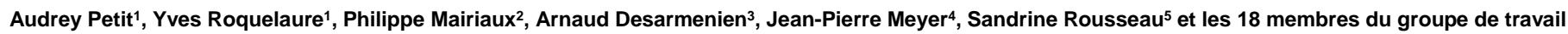

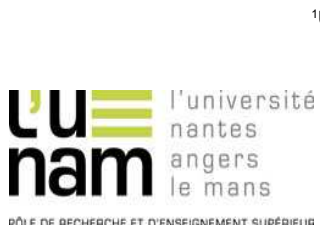

'Université LUNAM, Laboratoire d'Épidémiologie et d'Étude en Santé au Travail (LEEST), Université d'Angers, Faculté de Médecine d'Angers, France et Centre de Consultations de Pathologie Professionnelle, CHU Angers, France

${ }^{2}$ Service de Santé au Travail et d'Éducation pour la Santé, Université de Liège, Belgique

Service des Intervenants en Prévention des Risques Professionnels en Santé au Travail 72, Le Mans, France

${ }^{4}$ INRS, Centre de Lorraine, France

5Comité d'Hygiène Inter Entreprises, Saran, France
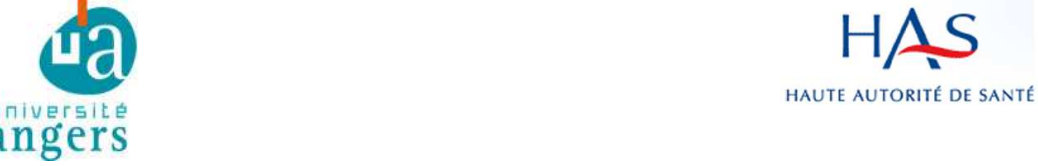

angers

\section{CONTEXTE}

Les manutentions manuelles de charges (MMC) sont ubiquitaires dans le monde du travail et concernent près de $40 \%$ des travailleurs de manière régulière (1). Principale cause d'accident du travail, elles exposent en particulier au risque de pathologies lombaires qui peuvent, en cas de récidive / chronicité, compromettre le parcours professionnel des travailleurs. Ainsi, près d'un tiers des travailleurs français souffrent de lombalgies dont $10 \%$ en souffrent quotidiennement.

La prévention des risques rachidiens liés aux MMC est donc un enjeu de santé au travail impliquant un très grand nombre d'entreprises de tout secteur d'activités et des situations de travail très variées. Elle doit s'intégrer dans le programme général de prévention des risques professionnels de l'entreprise (accidents, Troubles musculo-squelettiques, risques routiers, risques psychosociaux,...).

Cette communication résume les principales recommandations de bonne pratique pour la surveillance médico-professionnelle du risque lombaire pour les travailleurs exposés à des MMC parues en octobre 2013.
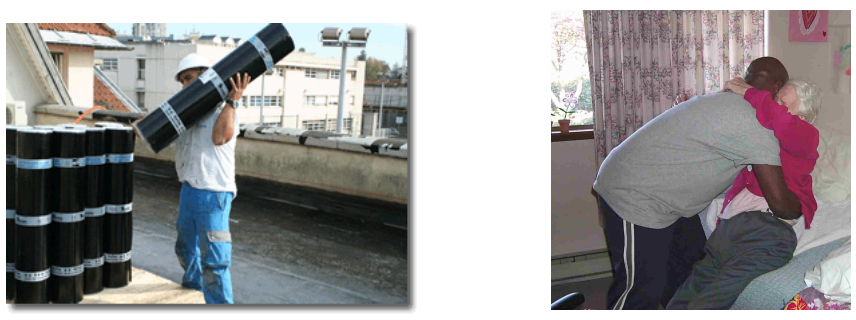

\section{SYNTHESE DE LA LITTERATURE}

\section{METHODE}

Ces recommandations de bonne pratique ont pour objectifs : exposition, travail. pluriprofessionnel de 50 relecteurs.

Tableau I. Professionnels concernés par les recommandations

d'améliorer le repérage et l'évaluation des situations professionnelles exposant à des manipulations de charges afin de limiter et/ou contrôler cette

- de définir la surveillance médicale adaptée afin de dépister et limiter les atteintes rachidiennes lombaires liées à l'exposition à des MMC dans le cadre d'une stratégie de prévention intégrée, collective et individuelle, en milieu de

Ces recommandations ont été élaborées selon la méthodologie de la Haute Autorité de Santé (HAS). La recherche documentaire (1990-2013) s'est étendue aux articles publiés, indexés ou non, à la littérature grise, aux recommandations françaises et internationales existantes, ainsi qu'aux rapports élaborés par les agences gouvernementales, les agences d'évaluation indépendantes et les sociétés savantes. Les banques de données biomédicales françaises et internationales, et de données spécifiques ont été interrogées. La synthèse de la littérature a été effectuée par un groupe de travail pluriprofessionnel de 24 experts. Une classification du niveau de preuve scientifique a permis la rédaction des recommandations validées par un groupe

Ces recommandations de bonne pratique s'adressent plus particulièrement aux médecins du travail qui animent une équipe pluridisciplinaire de santé au travail, mais également aux autres acteurs du système de soins (Tableau I).

\begin{tabular}{l|l|l|l|}
\hline Intervenants en santé au travail & $\begin{array}{l}\text { Intervenants du champ } \\
\text { des soins }\end{array}$ & $\begin{array}{l}\text { Intervenants du champ } \\
\text { médico-social }\end{array}$ \\
\hline SST & entreprises & Méd. du travail, & $\begin{array}{l}\text { IPRP, } \\
\text { IDEST }\end{array}$ \\
& $\begin{array}{l}\text { Ergonomes, } \\
\text { Psychologues,... }\end{array}$ & $\begin{array}{l}\text { Méd. Traitant, } \\
\text { Méd. Spécialistes, } \\
\text { Kinésithérapeutes, } \\
\text { Infirmiers, } \\
\text { Ergothérapeutes,... }\end{array}$ & $\begin{array}{l}\text { Méd. Conseil, } \\
\text { Chargés d'insertion et de } \\
\text { maintien en emploi, } \\
\text { Psychologues, Assistantes } \\
\text { sociales,... }\end{array}$
\end{tabular}

La réduction des risques liés aux MMC à la source doit être l'objectif prioritaire, mais, compte tenu des effets parfois modestes des interventions, il est nécessaire de développer des actions de prévention intégrée visant à la fois à prévenir l'apparition des lombalgies et à réduire leurs conséquences (2). Ces dimensions de la prévention ne doivent pas être opposées mais être intégrées dans une démarche de prévention globale.

En conséquence, la prévention du risque rachidien lié aux MMC doit intégrer des niveaux complémentaires visant de manière conjointe à

- limiter l'incidence des lombalgies chez les travailleurs exposés aux MMC (prévention primaire proprement dite) ;

- faire en sorte que ceux qui en souffrent déjà ne s'aggravent pas (prévention secondaire) ;

et puissent poursuivre leur activité professionnelle et se maintenir dans l'emploi de manière durable (prévention tertiaire).

Ainsi, même dans les situations où il est difficile de mettre en place des interventions primaires efficaces, il est le plus souvent possible de réduire la sévérité des cas et d'améliorer leur pronostic par des actions de prévention intégrée portant sur tous les stades de la lombalgie et de l'exposition aux MMC.

a prévention collective intégrée du risque rachidien liée aux MMC nécessite la coordination des prises en charge médico-sociales avec les actions sur le milieu de travail (3). Pour cela, elle doit être inscrite dans la politique de gestion des risques professionnels de l'entreprise afin de coordonner les actions et de les planifier en fonction de l'évaluation des risques.

La prévention globale des risques liés aux MMC doit être déclinée à l'échelle de l'entreprise, mais aussi (et surtout) pour les très petites entreprises et nombre de petites et moyennes entreprises, à l'échelle de la branche professionnelle ou des entreprises du même secteur d'activité suivies par le service de santé au travail. C'est un enjeu majeur de prévention qui nécessite l'établissement d'une politique de santé au travail permettant la hiérarchisation et la coordination des actions à l'échelle du territoire de santé ou de la branche professionnelle.

\section{RECOMMANDATIONS DE BONNE PRATIQUE}

La prévention du risque rachidien liée aux MMC doit s'inscrire dans la politique de gestion des risques professionnels de l'entreprise afin de coordonner et de planifier les actions en fonction de l'évaluation des risques.

Pour les très petites entreprises, il est recommandé de décliner la prévention intégrée des risques liés aux MMC à l'échelle :

- de l'entreprise,

- des entreprises du même secteur d'activité suivies par le service de santé au travail,

- de la branche professionnelle. 